\title{
ON FIXED POINTS OF COMMUTING ANALYTIC FUNCTIONS ${ }^{1}$
}

\author{
ALLEN L. SHIELDS
}

In this note we show that if $f$ and $g$ map the unit disc $|z| \leqq 1$ in the complex plane into itself in a continuous manner, if they are analytic in the open disc, and if they commute $(f(g(z))=g(f(z))$ for all $z)$, then they have a common fixed point $\left(f\left(z_{0}\right)=z_{0}=g\left(z_{0}\right)\right)$. More generally, any commuting family of such functions has a common fixed point.

In 1954 Eldon Dyer raised the following question: If $f$ and $g$ are two continuous functions that map a closed interval on the real line into itself and commute, must they have a common fixed point? The same question was raised independently by the author in 1955 and by Lester Dubins in 1956. A more general question was posed by Isbell [2] in 1957. These questions have not been answered.

The author wishes to thank N. D. Kazarinoff for several helpful discussions of this material.

Let $G$ be a bounded connected open set in the plane and let $F_{G}$ denote the family of all those analytic functions in $G$ whose range is contained in $G(f(G) \subset G)$. With the topology of uniform convergence in compact subsets, $F_{G}$ becomes a metric space. The functions in $F_{G}$ are uniformly bounded; hence [3, Chapter 2, \$7] each sequence of elements of $F_{G}$ contains a convergent subsequence (the limit function need not be in $F_{G}$ ).

Note that $F_{G}$ is a semigroup under composition of functions. The following lemma tells us that $F_{G}$ is a topological semigroup, that is, the semigroup operation is jointly continuous.

Lemma 1. If $f_{n} \rightarrow f, g_{n} \rightarrow g\left(f_{n}, f, g_{n}, g \in F_{G}\right)$, then $f_{n}\left(g_{n}\right) \rightarrow f(g)$.

Proof. Let $K$ be a compact subset of $G$ and let $U$ be a neighborhood of $g(K)$ whose closure $U^{-}$is compact and lies in $G$. Then $g_{n}(K) \subset U$ for large $n$. Also,

$$
\left|f(g(z))-f_{n}\left(g_{n}(z)\right)\right| \leqq\left|f(g(z))-f\left(g_{n}(z)\right)\right|+\left|f\left(g_{n}(z)\right)-f_{n}\left(g_{n}(z)\right)\right| \text {. }
$$

Let $z \in K$. Since $g_{n} \rightarrow g$ uniformly on $K$ and $f$ is uniformly continuous on $U^{-}$, the first term on the right is small when $n$ is large. Since $f_{n} \rightarrow f$ uniformly on $U^{-}$, the second term is also small.

Received by the editors May 16, 1963.

1 This work was supported in part by the National Science Foundation. 
Lemma 2. If $e(z) \in F_{G}$ is an idempotent, then either $e(z) \equiv z$ or $e(z)$ $=$ const.

Proof. Let $G_{1}=e(G)$. Since $e(e(z))=e(z)$, we have $e(z)=z$ for all $z \in G_{1}$. If $e(z)$ is not a constant, then $G_{1}$ is an open set; hence $e(z) \equiv z$. Q.E.D.

We require a few facts about compact semigroups which we state without proof (see [4]).

Let $S$ be a compact semigroup, let $x \in S$, and let $\Gamma(x)$ be the closure of the set of powers of $x$ :

$$
\Gamma(x)=\left\{x, x^{2}, \cdots\right\}-
$$

Then $\Gamma(x)$ is an abelian subsemigroup of $S$ and:

(i) $\Gamma(x)$ contains exactly one idempotent, $e$;

(ii) if $e$ is an identity for $\Gamma(x)$ then $\Gamma(x)$ is a group and $x$ has an inverse in $\Gamma(x)$;

(iii) if $e$ is a zero for $\Gamma(x)$ (that is, $e y=e$ for all $y \in \Gamma(x)$, then $x^{n} \rightarrow e$.

We return now to our function-theoretic situation. If $f \in F_{G}$ we denote the iterates of $f$ by $f_{n}$ :

$$
f_{1}=f, \quad f_{n+1}=f\left(f_{n}\right) \quad(n=2,3, \cdots) .
$$

LEMMA 3. Let $f \in F_{G}$ and assume that $f$ is not a homeomorphism of $G$ onto itself. Then there is a point $z_{0}$ in the closure of $G$ and there is a sequence $\left\{n_{i}\right\}$ of positive integers such that

$$
f_{n_{i}}(z) \rightarrow z_{0}
$$

uniformly on compact subsets of $G$.

Lemma 4. Same hypotheses. The point $z_{0}$ is a common fixed point for all continuous $g$ on $G^{-}$(the closure of $G$ ) that map $G$ into itself and commute with $f$ ( $g$ need not be analytic).

Proof. Fix $z \in G$. By Lemma 3,

$$
g\left(z_{0}\right)=g\left(\lim f_{n_{i}}(z)\right)=\lim g\left(f_{n_{i}}(z)\right)=\lim f_{n_{i}}(g(z))=z_{0} .
$$

Proof of Lemma 3. Let $\Gamma(f)$ denote the closure of the set of iterates of $f$ in the topology of uniform convergence on compact subsets of $G$.

Case I. $\Gamma(f) \subset F_{G}$. Then $\Gamma(f)$ is a compact semigroup and contains an idempotent $e(z)$. Since $f$ does not have an inverse in $F_{G}, e(z) \equiv z$ is impossible. Hence $e(z) \equiv z_{0} \in G$. This means that $e(z)$ is a zero for $\Gamma(f)$ and so $f_{n} \rightarrow z_{0}$.

Case II. There is a function $g \in \Gamma(f)$ not in $F_{G}$. Since $g$ is a limit of 
functions that map $G$ into itself we have $g(G) \subset G^{-}$. But $g \notin F_{G}$ and so there is a point $z^{\prime} \in G$ such that $z_{0}=g\left(z^{\prime}\right) \notin G$.

We claim that $g(z) \equiv z_{0}$. If not, then by Hurwitz's theorem [3, Chapter III, §11] given any neighborhood $U$ of $z^{\prime}$, one of the iterates of $f$ must take the value $z_{0}$ somewhere in $U$. But this contradicts the fact that the iterates of $g$ map $G$ into itself. Q.E.D.

For the case of the unit disc $D(|z|<1)$ a stronger result was proven in 1926 by Wolff [5] and Denjoy [1]. They showed that if $f$ is analytic in $D, f(D) \subset D$, then either $f$ is a bilinear map of $D$ onto itself having exactly one fixed point in $D$, or $f_{n} \rightarrow c$ for some constant $c(|c| \leqq 1)$. In the generality of Lemma 3 it seems unlikely that the full sequence of iterates will always converge to a constant.

If we include the "improper" point $z=\infty$ then Lemma 3 can be strengthened. If $G$ is any connected open set on the Riemann sphere whose complement contains at least three points, then the conclusion of the lemma is valid. Here one needs a theorem of Montel [3, Chapter $7, \S 13]$ stating that a family of meromorphic functions that omits three values is a normal family.

We need a result about the fixed points of analytic homeomorphisms of the disc $D$ onto itself. This is not new but we could not find it stated explicitly. We include the proof for completeness.

Lemma 5. Let $f$ be a bilinear map of $D$ onto itself. Then three cases are possible:

(i) $f=z$;

(ii) $f$ has exactly one fixed point in the closed disc;

(iii) $f$ has two distinct fixed points on the boundary of $D$ and the iterates of $f$ converge to one of these points.

Proof. The general form for $f$ is

$$
f(z)=\alpha \frac{z-a}{1-\bar{a} z}, \quad|\alpha|=1, \quad|a|<1 .
$$

Assume that $f \neq z$. From the equation $z=f(z)$ for fixed points we have

$$
\bar{a} z^{2}-(1-\alpha) z-\alpha a=0 .
$$

This equation is unchanged if $z$ is replaced by $1 / \bar{z}$. Hence the fixed points of $f$ are inverse to one another with respect to the unit circle. So either there is one point inside the circle and one outside, or there is one "double" fixed point on the boundary, or there are two distinct fixed points on the boundary.

Only the last case needs to be considered. Let $p$ be a bilinear map 
taking $D$ onto the upper half-plane and taking the two fixed points into 0 and $\infty$. Let $g=p f p^{-1}$. Then 0 and $\infty$ are fixed points of $g$, and $g$ maps the upper half plane onto itself. Hence $g(z)=a z(a>0)$. Also, $a \neq 1$ since $f \neq z$. The iterates of $g, g_{n}(z)=a^{n} z$, converge either to 0 or to $\infty$, hence the iterates of $f$ converge to one of the two fixed points of $f$. Q.E.D.

THEOREM 1. Let $F$ be a commuting family of continuous functions mapping the closed unit disc into itself and analytic in the open disc $D$. Then there is a common fixed point $z_{0}$ for all the functions of the family.

Proof. If $F$ contains a constant function, this is our fixed point. If not, then each function maps $D$ into itself, by the maximum modulus theorem. Hence Lemma 4 can be applied, unless all the functions are bilinear maps of $D$ onto itself. In this case Lemma 5 applies (if a function has just one fixed point it is a fixed point for all functions commuting with the given function). Q.E.D.

\section{BIBLIOGRAPHY}

1. A. Denjoy, Sur l'itêration des fonctions analytiques, C. R. Acad. Sci. Paris 182 (1926), 255-257.

2. J. R. Isbell, Commuting mappings of trees, Research Problem 7, Bull. Amer. Math. Soc. 63 (1957), 419.

3. S. Saks and A. Zygmund, Analytic functions, Monogr. Mat., Vol. 27, Polsk. Towarzystivo Mat., Warsaw, 1952.

4. A. D. Wallace, The structure of topological semigroups, Bull. Amer. Math. Soc. 61 (1955), 95-112.

5. J. Wolff, Sur l'itêration des fonctions holomorphe ..., C. R. Acad. Sci. Paris 182 (1926), 42-43, 200-201.

UNIVERSITY OF MICHIGAN 